\title{
KRIMTATARISCHE SOLDATENLIEDER AUS DEM ERSTEN WELTKRIEG
}

\author{
ZSUZSA KAKUK* \\ (Budapest)
}

\begin{abstract}
Crimean Tatars were detained in a prison camp near the Hungarian town Esztergom during World War I. Ignác Kúnos collected rich linguistic and folklor data from them. The paper publishes a selection of this material. The texts four liners and parts from longer poems recall their grievance due to the solder's life, war and camp life.
\end{abstract}

Key words: Crimean Tatars, folklore, Ignác Kúnos.

Das Schicksal - sei es auch so schwer zu erleiden - kann unser Leben manchmal auch günstig beeinflussen. Das geschah auch Lajos Fekete: er geriet als Soldat während des ersten Weltkrieges in Kriegsgefangenschaft, er wurde nach Sibirien gebracht und dort lernte er von seinen türkischen Mitgefangenen Türkisch. Die auf diese Weise erworbenen Sprachkenntnisse determinierten seine Zukunft: in Ungarn heimgekehrt fing er an, die Zeit der Türkenherrschaft zu erforschen. Er hat die türkischen Sprachdenkmäler der Zeit immer gründlicher kennengelernt und gründete eine äußerst bedeutende historische Disziplin: die türkische Paläographie.

In denselben Kriegsjahren kamen russische Gefangene - unter ihnen Männer aus verschiedenen türkisch sprachigen Ländern - in die auf dem Gebiet der Österreichisch-Ungarischen Monarchie gebauten Lager. Neben der tschechischen Stadt Eger (dem heutigen Cheb) und dem ungarischen Esztergom wurden Sonderlager für Muslime errichtet. In den vier Jahren des Krieges besuchte der ungarische Turkologe Ignác Kúnos mehrmals diese Lager und sammelte tatarisches Folklormaterial in großem Umfang. Im Lager in Eger sammelte er das kasantatarische und das mischärtatarische Material, das krimtatarische sammelte er im Lager in Esztergom. ${ }^{1}$

${ }^{*}$ Zsuzsa Kakuk, H-1022 Budapest, Csopaki u. 3/A, Hungary.

${ }^{1}$ Der größte Teil des Materials ist bereits verlegt worden.

Ich plane das kasantatarische Material in drei Bänden. 1. Volkslieder, 2. Volksmärchen, 3. Wörterbuch. Davon sind die Bände der Volkslieder und der Volksmärchen schon erschienen, an der Ausgabe des Wörterbuches wird noch gearbeitet: Kasantatarische Volkslieder. Auf Grund der Sammlung von Ignác Kúnos herausgegeben von Zsuzsa Kakuk. Budapest. 1980 (Oriental Studies No. 4); Kasantatarische Volksmärchen. Auf Grund der Sammlung von Ignác Kúnos, herausgegeben 
Im folgenden Artikel habe ich dem krimtatarischen Material Vierzeiler und Zitate aus längeren Gedichten entnommen, die die oft erschütternden Erlebnisse des Soldatenlebens, des Krieges und der Gefangenschaft widerspiegeln.

Am Anfang scheint das Soldatenleben überhaupt nicht so schwierig zu sein: Heiter verabschieden sich die Jungen vom Vater, von der Mutter, der Geliebten, von den Freunden und vom Zuhause. Sie sind sogar stolz darauf, Soldat zu sein - sie sind ja auch an ihrem Gang zu erkennen, zu Hause heimgekehrt wird sie die zierliche Geliebte erwarten.

1. (292. Mâni)

Kükte yıldız ellidir ellisi de bellidir asker bolğan yigetni yürüşinden bellidir

2. (95. Mâni)

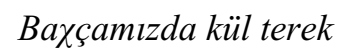
kül terekke su kerek saldat kaytkan akamğa ince belli kız kerek

3. (Zitat aus dem 62. Türkü)

Davul zurna çaldırdım keçtim buradan sauluknan kal anacı̆̆ım kaytırmen yuldan

Kitecekmiz küyümden kal sauluknen sil küzinin yaşını al yauluknen

\author{
Sterne am Himmel - es gibt gerade fünfzig \\ alle sind von hier zu sehen \\ der junge Soldat \\ ist an seinem Gange zu erkennen.
}

In unserem Garten steht ein Rosenbaum der Baum braucht Wasser mein zurückkehrend Bruder braucht ein zärtliches Mädel

Ich ließ mir Davul, Zurna spielen dann ging von hier weg leb wohl, Mütterchen ich werde zurückkehren

Wir verlassen jetzt unser Dorf leb wohl! wisch die Tränen vom Auge mit deinem roten Tuch

von Zsuzsa Kakuk und Imre Baski. Budapest. 1989 (Oriental Studies No. 8); Kasantatarisches Wörterverzeichnis. Auf Grund der Sammlung von Ignác Kúnos herausgegeben von Zsuzsa Kakuk und Imre Baski (unter Bearbeitung).

Das krimtatarische Material wird ebenso aus drei Bänden bestehen. Davon ist nur ein Band erschienen: Kırım Tatar Sarkıları. I. Kúnos'un derlemesinden yayımlayan Zsuzsa Kakuk. Ankara. 1993 (Türk Dil Kurumu Yayınlar1: 564). In einer längeren Studie habe ich mich ausführlich mit den krimtatarischen Rätseln beschäftigt, die am Ende der Volksliedersammlung zu finden sind: Zsuzsa Kakuk: Krimtatarische Rätsel. (Esztergom 1915). In: Acta Orientalia Hungarica XLVII. 1994. ff. 143-173. Die Volksmärchen werden noch bearbeitet, Imre Baski arbeitet daran. Am Wörterbuch arbeiten wir zusammen. - Als selbständiger Band ist das mischärtatarische Material erschienen, der Lieder, Märchen und das Wortmaterial beinhaltet: Zsuzsa Kakuk: Mischärtatarische Texte mit Wörterverzeichnis. Szeged, 1996 (Studia Uralo-Altaica 38). 
4. (Zitat aus dem 12. Türkü)

Ak-mecid yull çakıl taş

atı da nallı at

blyllkı yaşım un sekiz

üç yıldan saldat

Portokalnı uydurdim içine güller kuydurdım şu mâlede br yar süydim anasina tuydurdim
Die Straßen von Akmetschit sind gepflastert auch deinem Pferd ist die Hufe beschlagen dieses Jahr wurde ich achtzehn drei Jahre werde ich Soldat (sein)

Die Orange habe ich ausgehöhlt und eine Rose darin gesteckt im Viertel habe ich eine lieb ihre Mutter ließ ich's wissen

Aber in der Kaserne erwartet die jungen Soldaten ein schwierigeres Schicksal: der tatarische Kalpak wird ihnen weggenommen, sie bekommen Soldatenmütze, die Befehlshaber verhalten sich grob mit ihnen, die Ausbildung ist schwer, sie müssen schwere Prüfungen überstehen. Aber noch schwieriger ist die Sehnsucht nach der Geliebten, nach dem Zuhause zu vertragen.

5. (39. Mâni)

Anem dedim anem yok babam dedim babam yok kazarmă̆a hasta düştim nedir alı degen yok

6. (97. Mâni)

Bahçasaray kan-saray unda yattum bir talay stavay saldat degende küz yaşını tıyalmay

7. (Zitat aus dem 45. Türkü)

Aşadan keliy pristavlar aklimı al̆̆an başımdaki kalpağım alıp şapkasin salğan

Azbarımı bekley bekley çalısı kelecek anemden gayrı kimsem yok avladim deyecek

Azbarımı bekley bekley çalınen kazık asker ketkenime yanmayım aneme yazı
Mutter - sagte ich, sie ist aber nicht hier Vater - sagte ich, er ist aber nicht hier in der Kaserne wurde ich krank Wie geht es dir? - fragte aber niemand

Serail der Bachtschiserail-Khane da verweilte ich eine Weile Auf! Soldat auf! - sagten sie die Tränen konnte man nicht unterdrücken

Die Werber kommen von unten ich habe meinen Verstand fast verloren sie nahmen mir den Kalpak weg und warfen mir die Soldatenmütze zu

Unser Hof wartet und wartet das Gebüsch kommt hervor ich habe niemand außer meiner Mutter die mir sagen würde - mein Sohn!

Unser Hof wartet und wartet der Köder und der Distel daß ich Soldat geworden, gereue ich nicht es tut mir leid allein für meine Mutter 


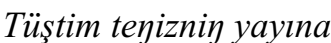
sayladım daşı ah aneyim să̆ındım pişirgen aşnı
Ich kam an die Küste des Meeres und zählte die Steine Ah, Mutter, wie oft dachte ich an deine Kost

Dann kam die wirkliche Not: der Krieg brach aus. Die jungen Männer rücken nicht nur ein, sie gehen in den Krieg. Sie kommen immer weiter von der Heimat, von Zuhause weg.

\section{8. (98. Türkü)}

Kazarmanıท dübi daşdır küzimizden axan yaşdır gurbetligi çeken başdır aman Allah, imdad eyle

Kazarmanıy merdimeni ne müşü̈ldür derdim beni

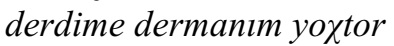

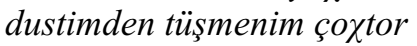

Kazarmalar buydan buya men tüşkenim bir tar yola yulım ayrılığa tüşken aman Allah, imdad eyle

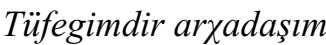
ă̆lap tüktim küzim yaşın yoktor anam, yoktor babam

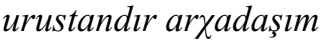

Kazarma üginde tirek ăglayım tayanmay yürek bunda bizge mal ne kerek kiyâmet bar ne eylenek

9. (53. Türkü)

Kiteyim kiteyim yulım tükenmey tüneyim baxayım silam körülmiy Tuttırdım kadeyi zeher içilmey asretlik gurbetlik is te çekilmey
Die Kaserne ist vorne gepflastert Tränen fließen aus unseren Augen In-der-Fremde-sein lastet auf uns Ach, Allah, hilf uns!

Wie die Treppe der Kaserne so groß ist meine Not sie ist nicht $\mathrm{zu}$ heilen ich habe mehr Feinde als Freunde

Es gibt nur Kasernen weit und breit mir ist die Not zuteil geworden mein Schicksal ist der Abschied Ach, Allah, hilf mir!

Mein Freund ist mein Gewehr Ich weine, Tränen strömen aus meinen Augen ich habe weder eine Mutter, noch einen Vater mein Kamerad ist von den Russen

Vor der Kaserne steht ein Pfahl mir tut das Herz weh, ich muß weinen was eigentlich bräuchten wir hier? das ist der Untergang, was können wir denn tun?

Ich laufe nur, ich laufe mein Weg hat kein Ende ich wende mich um, ich blicke zurück mein Zuhause ist nicht mehr zu sehen Ich nahm den Becher der Gift ist aber nicht zu trinken die Sehnsucht, der Abschied sind nicht zu ertragen 
Ey akalar büyle zulum bolor-mo ecel kelmeyince ülüm bolor-mo Kapımızıฤ ögi kayall daşlı ey garib anemin güzleri yaşlı

Aldım tüfegimi çıtım tâlimge benden selam bolson garib anemge U çayırlar kalğan paydası kalğan aneler babalar balasız kalğan

10. (Zitat aus dem 70. Türkü)

Kırımın çevresi bustan men ayrildım eşten dusttan endi çı $\chi$ tı yurdumuzdan eman Mevlâm kutar bizi

Amayl taxsın kuynıma ecelni aldım buynıma tüştim islâm yulına eman Mevlâm kutar bizi
Ei, mein Bruder, wieso gibt es diese Unterdrückung? Bevor der Engel des Todes kommt, können wir sterben? Vor unserem Tor gibt es felsigen Boden, Steine Ei, meine verlassene Mutter hat Tränen in den Augen

Ich nahm mein Gewehr ging auf die Feldübung sei gegrüßt, meine verlassene Mutter! Die Äcker liegen brach sie haben keinen Ertrag die Väter, die Mütter haben keine Kinder mehr

Die Krim-Gegend ist voller Gärten ich schied von den Kameraden, Freunden ich verließ meine Heimat Ach, mein Gott, befrei uns!

Man soll ein Amulett an meine Brust hängen ich nahm den Tod auf mich ich kam auf den Weg des Islam Ach, mein Gott, befrei uns! dentod.

Auf dem Schlachtfeld erwarten sie viel Leiden, Verletzungen und der Hel-

\section{1. (Zitat aus dem 131. Türkü)}

Kün tutuld k kündüz tüngen yoxsen zaman ahri bolğan padişalar ayakka turğan tabır-asker çöle sürgen

Asker leşle çöller tol̆gan soğışdan çıkkan yaralı bolğan şok balalar yetim bolğan ümürlere peşmân bolğan
Die Sonne wurde finster, der Tag wurde Nacht es kam vielleicht das Ende der Zeiten die Padischachs sprangen alle auf das Lager, die Soldaten zogen aufs Schlachtfeld Die Felder sind mit den Leichen der Soldaten voll die Heimkehrenden wurden verletzt viele Kinder sind verwaist sie bereuten es, geboren zu sein 
Biz tüşmânğa karşı barğan tüşmanlarımız kırarğan Allah için kurban bolğan cennetge kirmege yul bol̆̆an

Şehit bolğan cennetke kirey Akkın Resulın anda küriy

12. (127. Türkü)

\author{
Şok balalar şeyit bolğan \\ şok babalar ülep bolğan \\ sizinnen de kürişerek \\ mahşere kalğan \\ şeyitler için \\ duha kil̆gan
}

Bizim işin milletimiz duha kılıy mı bizden sonki balalarıy yüzleri küley-mi sabr etip kunğannar külmek bilmegenler

Biz ăglarmı biz cılarmı yârim yârim deye yâr ă̆layır, yâr cılayır erim erim deye gece kündüz ă̆larmı yârim erim deye

Başları tüşken gül kibik sunğanlar bek kübisi bu âlinen şeyit bolğanlar
Wir zogen gegen den Feind wir besiegten ihn wir opferten unser Leben für Allah wir fanden den Weg, der in den Himmel führt

Der Gefallene kommt in den Himmel er erblickt den Gott und den Propheten
Es gab viele Junge, die gefallen sind es gab viele Väter, die gestorben sind die Begegnung mit Euch wird im Jenseits stattfinden für die Gefallenen sprachen wir ein Gebet

Wird wohl unser Volk für uns ein Gebet sprechen?

kann das Gesicht unserer Kinder noch lachen? sie warten geduldig sie können nicht einmal lachen

Wir weinen, wir schluchzen mein Liebling, mein Liebling - sagen wir die Geliebte weint, sie schluchzt mein Mann, mein Mann - sagt sie wir weinen Tag und Nacht mein Liebling, mein Mann - sagen wir

Ihre Köpfe sanken wie die Rose wurden sie gelb sehr viele von ihnen fielen unter solchen Umständen

Das Schönste von den Liedern über den Heldentod ist einfach, aber spricht mit tiefem Gefühl über diese Art des Sterbens.

13. (Zitat aus dem 45. Türkü)
Biz bu yerge ülersek kim yuvay bizni yăgmur yavay seller akay sel yuvay bizni

Sterben wir hier im Fremden wer wäscht unsere Leichen? es regnet, es weht der Wind wäscht uns 
Biz bu yerge ülersek

kim kümey bizni

bahar ulsa utlar kitey

ut kümey bizni
Sterben wir hier im Fremden wer begräbt unsere Leichen? der Frühling wird kommen, Gras wächst das Gras wird uns begraben

In den Liedern über die Schlachten und über die Gefangenschaft tauchen immer öfter die Ortsnamen auf, wo die Schlachten stattfinden: Kiev, Lvov, Galizien, die Karpaten; dann die Orte der Gefangenschaft: Ungarn, Österreich, und schließlich die Donau und Esztergom.

14. (Zitat aus dem 89. Türkü)

Akmeçitten çıktım Kyevağa keldim

Kyevada turmayıp vaynağa keldim

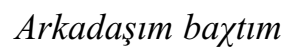
kurşun yarası uta yansin Afstriyanı Karpat gorasi

15. (Zitat aus dem 14. Türkü)

Azırlağan maşınamız birbirine bağlağan eger işler büyle ketse cümle alem cllağan

Talerkadan yüzüm aldım avıştırdım tabă̆a Galitsyanıy suvaşında bel boğazdan kan aka

16. (29. Türkü)

Polkovniktir başımız

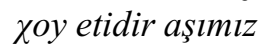
şu Karpatnıฑ içinde kaldı cümle yaşımız

Bizni pilen alğanlar maşınă̆a salğanlar bizinnen arkadaşlar cümle ă̆lap kalğanlar
Ich kam aus Akmetschit weg ich kam in Kiev an ich hielt nicht einmal an ich ging gleich in die Schlacht

Ich sah meinen Kameraden an die Wunde einer Kugel an ihm die Berge Österreichs und der Karpaten sollen im Feuer untergehen

Die Waggons wurden vorbereitet aneinander geschlossen wenn es weiter so geht die ganze Welt wird elendig sein

Ich nahm von der Schüssel Trauben legte sie in meinen Teller in den Schlachten in Galizien fließt das Blut vom Hals und Leib

Der Oberst ist unser Kommandant Schaffleisch essen wir in den Karpaten bleiben all unsere Jungen

Wir sind jetzt gefangen in die Waggons gestellt mit unseren Kameraden haben wir viel geweint 
17. (65. Türkü)

Şu Karpatnıり taularında bizge akup kazılğan elbet büyle kürecekmiz başımız̆̆a yazıl̆̆an

Başımızda yeşil şapkı al kuşağım băgladım Istirgumnin barakların dürt aylanıp ağladım

Elimdegi yaulı̆̆ımnı işleteyim dürt uçın salâm süleyiz yârime ağlamasın ben için
In den Bergen der Karpaten grub man uns eine Schanze wir werden jetzt wohl erfahren was für ein Geschick uns zuteil wird

Die grüne Mütze auf unserem Kopf ich band meinen roten Gürtel um die Baracken in Esztergom umging ich weinend viermal

Das Tuch an meiner Hand ist an den vier Ecken bestickt meine Grüße an meine Geliebte sie soll nicht um mich weinen!

Unter den besseren Bedingungen des Esztergomer Gefangenenlagers kommt nicht weniger intensiv, aber mit einer heitereren Stimmung die Hoffnung auf die Heimkehr auf. Sie bemerken sogar die schönen Mädchen in Esztergom.

18. (Zitat aus dem 28. Türkü)
Maşınalar yă̆ladık birbirine bağladık Galitsyada alındık Estirgumda saylandik
Filiz fidan buyımız ağlap kalğan suyımız Macaryadan kaytkan soy bolor bizim tuyımız

19. (132. Mâni)

\section{Bukün cuma ertesi Estergumnin bazarl gezey çarşularında güzellerniy nazarl}

20. (317. Mâni)
Mindigim takta parçası kittigim Tunay arası endi Girman bik yaman bulunmağan çârası

Die Waggons behandelten wir mit Fett und zusammengekoppelt in Galizien wurden wir gefangen in Esztergom gezählt

Zarter Sproß ist unser Leib unsere Familie weint zu Hause aus dem Ungarnland heimgekehrt werden wir die Hochzeit haben

Heute haben wir Samstag in Esztergom gibt es Markt an den Ständen geht der Blick der schönen Mädchen hin und her

Ich ging in den Hafen ich wanderte im Donauland der Deutsche ist ja böse es gibt kein Mittel gegen ihn 
21. (308. Mâni)

Mektüp yazdım otorlp hoş salamnar tottorlp mektüp kitken men kal̆gan Avstriyada otorip

22. (Zitat aus dem 14. Türkü)

Kamançemi ă̆acı eriktendir erikten ben naslı ayrlayım senin kibik ferikten

Kemi keliy Tunadan tutulğan firtınadan elinde kelin-kuman kiyattaki kinadan

Duvar üstünde turdım turasiz para buldim bar kit Emine Şerife senden yaxşı yâr buldım
Ich sitze hier, ich schrieb einen Brief ich schickte Grüße an alle der Brief ist weg, ich aber blieb hier ich sitze hier in Österreich

Mein kemençe ist aus Holz aus Pflaumenholz, aus Pflaumenholz wie soll ich mich denn verabschieden von einem Wächtelchen wie dir

Ein Schiff kommt auf der Donau ein Sturm hat ihn erwischt in ihrer Hand der Hochzeitskrug Henna im Papier

Ich saß auf der Mauer ich fand eine Mütze ohne Prägung geh ruhig Emine Şerife ich fand eine bessere Liebhaberin 